\title{
Photonic Band Structure of Fibonacci Superlattices with Metamaterials. Part 1: The Algebraic Method of Calculation for Lossless Layers
}

\author{
Karol Tarnowski, ${ }^{*}$ Włodzimierz Salejda \\ Institute of Physics, Faculty of Fundament Problems of Technology, Wroclaw University of Technology, \\ Wybrzeże Wyspiańskiego 27, 50-386 Wrocław
}

Received August 31, 2009; accepted September 17, 2009; published September 30, 2009

\begin{abstract}
We study the properties of the photonic band structure (PBS) of infinite and binary Fibonacci superlattices (FS) containing metamaterial lossless layers. The transfer matrix method (TMM) and periodic boundary conditions are applied. The algebraic method of PBS calculations, based on the Bloch theorem, is described and used. The dispersion relations, for both type of light polarization, characterizing peculiarities of PBS are calculated numerically and presented. Fractal properties of a photonic band structure and the existence of so called zero- $\bar{n}$ gaps are established.
\end{abstract}

The physical properties of low dimensional aperiodic (translationally ordered) systems have attracted significant attention in recent years [1,2]. In particular, the PBS and transmission of light through periodic and aperiodic multilayers have been studied extensively [1-22 and references cited therein].

The main task of this short note (part I) is twofold. We shall present: 1) the preliminary results concerning the photonic band structure of FS with metamaterial slabs and 2) the algebraic method of PBS calculations.

The studied system is an infinite periodic approximant of Fibonacci superlattice $[15,16,18,20]$. The unit cell is composed of $J$ isotropic and homogeneous layers with refractive indices $n_{j}$ determined by their relative permittivities $\varepsilon_{j}$, permeabilities $\mu_{j}$ and thicknesses $d_{j}$, where the subscript $j$ denotes $j$ th layer.

The considered binary superlattice consists of $\mathbf{A}$ (nondispersive, right handed material, RHM) and $\mathbf{B}$ (dispersive, left handed material, LHM) slabs, with permittivity, permeability, and thickness which can take one of two sets of values: $\mu_{\mathbf{A}}, \varepsilon_{\mathbf{A}}$ and $d_{\mathbf{A}}$, or $\mu_{\mathbf{B}}, \varepsilon_{\mathbf{B}}$ and $d_{\mathbf{B}}$. The order of a layer in the unit cell is defined by substitution rules: $S_{0}=\mathbf{B}, S_{1}=\mathbf{A}, S_{L}=S_{L-1} \cdot S_{L-2}$, where $L=2,3, \ldots$, denotes the generation number and “•” means concatenation.

In the framework of the transfer matrix method, the translational invariance, according to the Bloch theorem, imposes on amplitudes of the electric field vectors on borders of the unit cell $\left(E_{\text {in }}^{+}, E_{\text {out }}{ }^{+}, E_{\text {in }}{ }^{-}, E_{\text {out }}{ }^{-}\right)$the following relation:

\footnotetext{
*E-mail: karol.tarnowski@pwr.wroc.pl
}

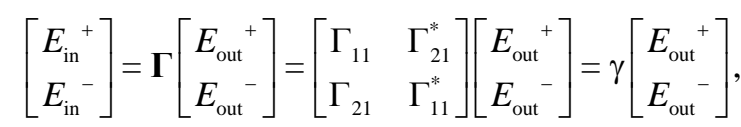

where $\boldsymbol{\Gamma}$ is the $2 \times 2$ characteristic unitary matrix (det $\Gamma=1$ ), with elements depending on parameters of the structure, described above, and parameters of the electromagnetic wave: the free space wavelength $\lambda$, the polarization ( $\mathrm{s}$ or $\mathrm{p}$ ), and the incidence angle $\theta$. The parameter $\gamma$ is the Bloch factor, i.e. eigenvalue of $\Gamma$ and $\gamma=\exp (-\mathrm{i} q d)$, where $q$ is the Bloch vector and $d$ is the unit cell thickness (length of $S_{L}$ ). Equation (1) is equivalent to:

$$
\operatorname{det}\left[\begin{array}{cc}
\Gamma_{11}-\gamma & \Gamma_{21}^{*} \\
\Gamma_{21} & \Gamma_{11}^{*}-\gamma
\end{array}\right]=1-\gamma \tau+\gamma^{2}=0,
$$

where $\tau$ is the trace of $\Gamma$ matrix and depends on all parameters defining FS and the electromagnetic wave.

Solving (3) we obtain the fundamental equation that our algebraic method is based on

$$
q d=\arccos \left(\frac{\tau}{2}\right)
$$

The proposed approach allows us to calculate PBS for superlattices containing: dispersive and nondispersive materials (in terms of permittivity and permeability), both right- and left- handed materials and periodic, aperiodic and random superlattices.

The presented algebraic method can be used to find from equation (3) the normalized Bloch vector $q$ in the first Brillouin zone, for the fixed frequency $\omega$ and the incidence angle $\theta$. This allows us to get 3D plot of $\omega(q, \theta)$ and dispersion relations: $\omega(q)$ for the fixed $\theta, \omega(\theta)$ for the fixed $q, \omega\left(k_{\perp}\right)$ for the fixed $q$, where $k_{\perp}=n \omega \sin (\theta) / c$ is the perpendicular component of the electromagnetic wave vector. 
Below we present PBS for the model of Fibonacci superlattices with the following slab materials:

A - the free space,

B - the metamaterial with material dispersion $[3,11]$ :

$$
\varepsilon(\omega)=1-\frac{\omega_{\mathrm{pe}}^{2}}{\omega^{2}}, \quad \mu(\omega)=1-\frac{F \omega^{2}}{\omega^{2}-\omega_{\mathrm{pm}}^{2}},
$$

where the following parameters are assumed: $\omega_{\mathrm{pe}}=15.1\left[10^{15} \mathrm{~Hz}\right], \quad \omega_{\mathrm{pm}}=2.39\left[10^{15} \mathrm{~Hz}\right], \quad \mathrm{F}=0.98$; which gives a negative value of both $\mu$ and $\varepsilon$ in frequency range of $\left(\omega_{\mathrm{pm}}, \omega_{\max }\right)$, where $\omega_{\max }=15.069 \cdot 10^{15} \mathrm{~Hz}$ corresponds to the free space wavelength $125 \mathrm{~nm}$. Plots of $\varepsilon(\omega), \mu(\omega), \mathrm{n}(\omega)$ are depicted in Fig. 1 .

The layer thickness is $d_{\mathbf{A}}=125 \mathrm{~nm}, d_{\mathbf{B}}=39.3 \mathrm{~nm}$, respectively, which gives the quarter-wave layers at a wavelength of $500 \mathrm{~nm}$.

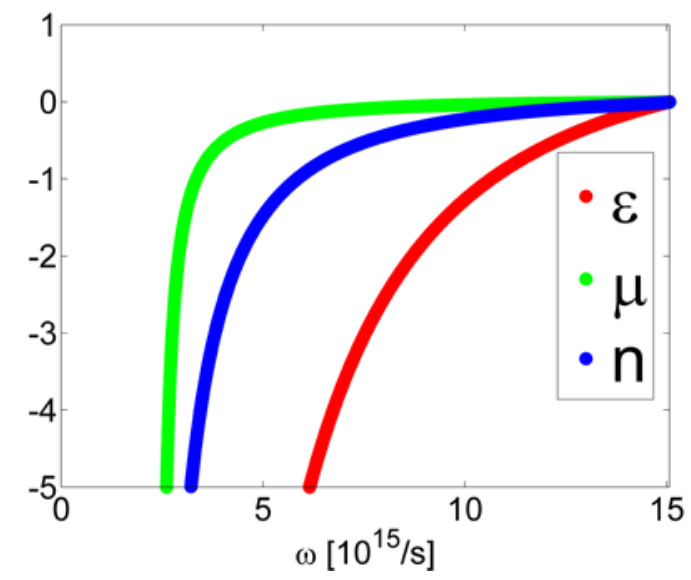

Fig. 1. Material dispersion of B (LHM) slab, permittivity - red, permeability - green, refractive index - blue

Figure 2 presents photonic the dispersion relation $\omega(q)$ for selected values of $\theta$. Polarizations s and $\mathrm{p}$ are denoted with blue and red colors, respectively. Results for the periodic approximant of $4^{\text {th }}$ generation of FS (ABAAB) are presented.

Figure 3 shows PBS of FS for different number of generations. The black and light grey area corresponds to the band gap and pass band in $\omega\left(k_{\perp} \sim \omega \sin (\theta) / \omega_{\max }\right)$, respectively. Moreover, the right- and left-hand halves contain the results for $s$ and p polarizations, respectively.

We want to underline existence of zero- $\bar{n}$ photonic band gaps [23], which positions in PBS depend on the thickness of layers and assumed material dispersion. For generations of FS presented in Fig. 3 these gaps are present in relatively wide ranges of incidence angle $\theta$ at the following frequency: $3.26 \cdot 10^{15} \mathrm{~Hz}$ for $4^{\text {th }}$, $3.16 \cdot 10^{15} \mathrm{~Hz}$ for $5^{\text {th }}, 3.20 \cdot 10^{15} \mathrm{~Hz}$ for $6^{\text {th }}, 3.18 \cdot 10^{15} \mathrm{~Hz}$ for $7^{\text {th }}$ generation.
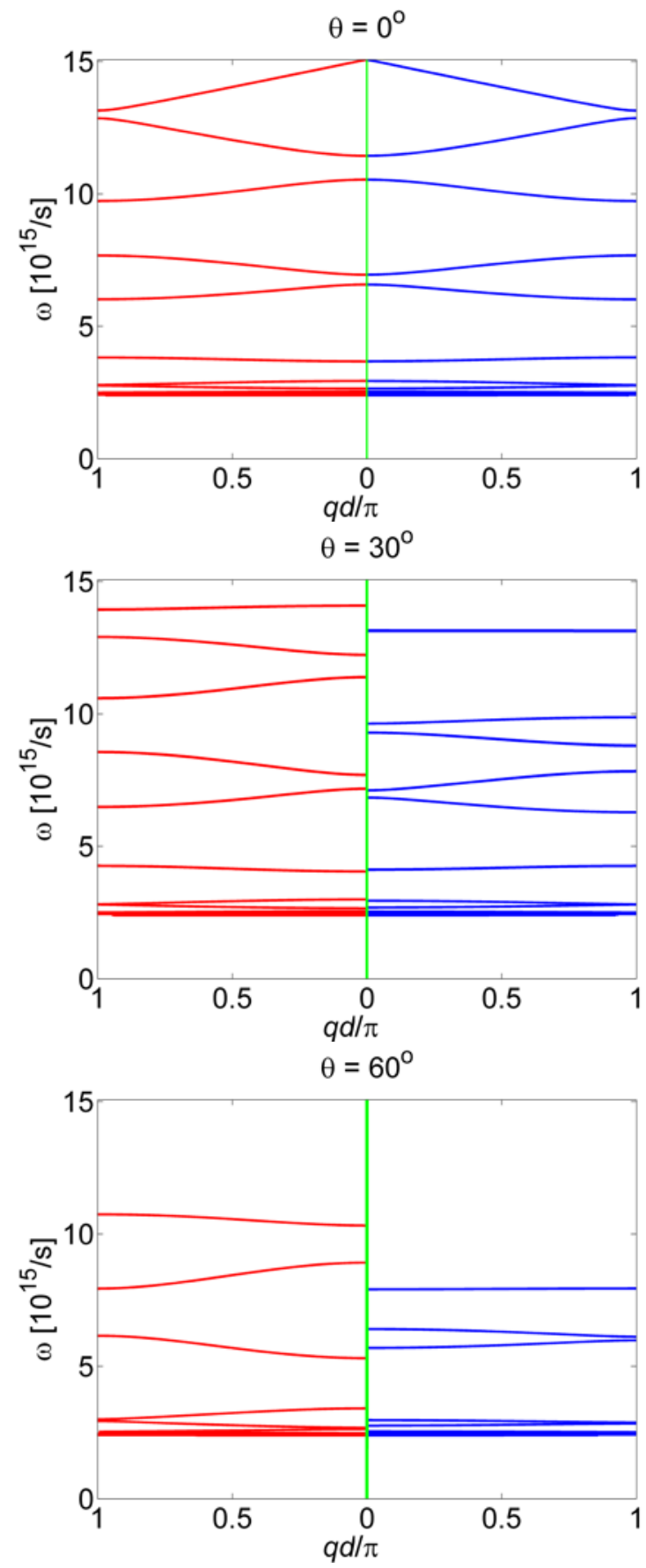

Fig. 2. Photonic dispersion relation $\omega(q)$ for $\theta=0^{\circ}, 30^{\circ}, 60^{\circ}$ for periodic approximant of Fibonacci superlattice (4th generation)

In summary, we presented the calculation method of a photonic band structure of infinite periodic and periodic approximants of translationally ordered aperiodic superlattices. This method is based on the transfer matrix method and the Bloch theorem and 
a)

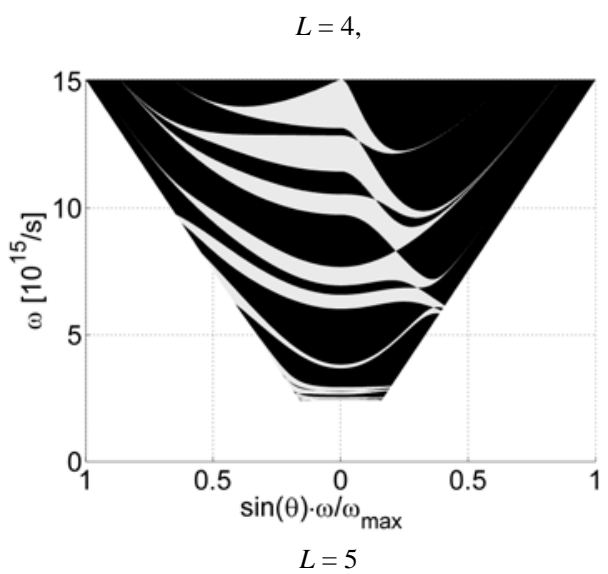

b)

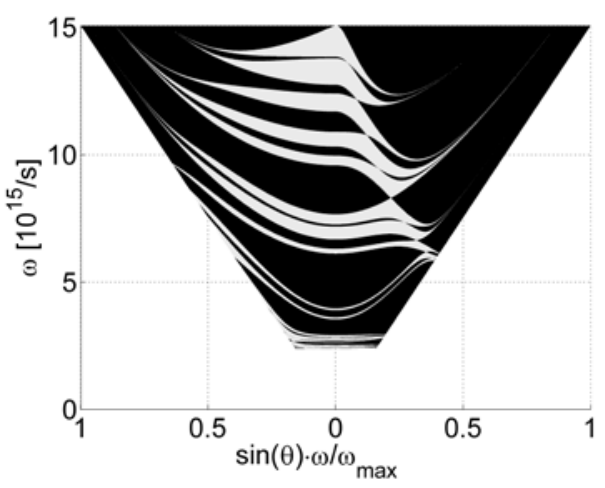

c)

$L=6$,

d)
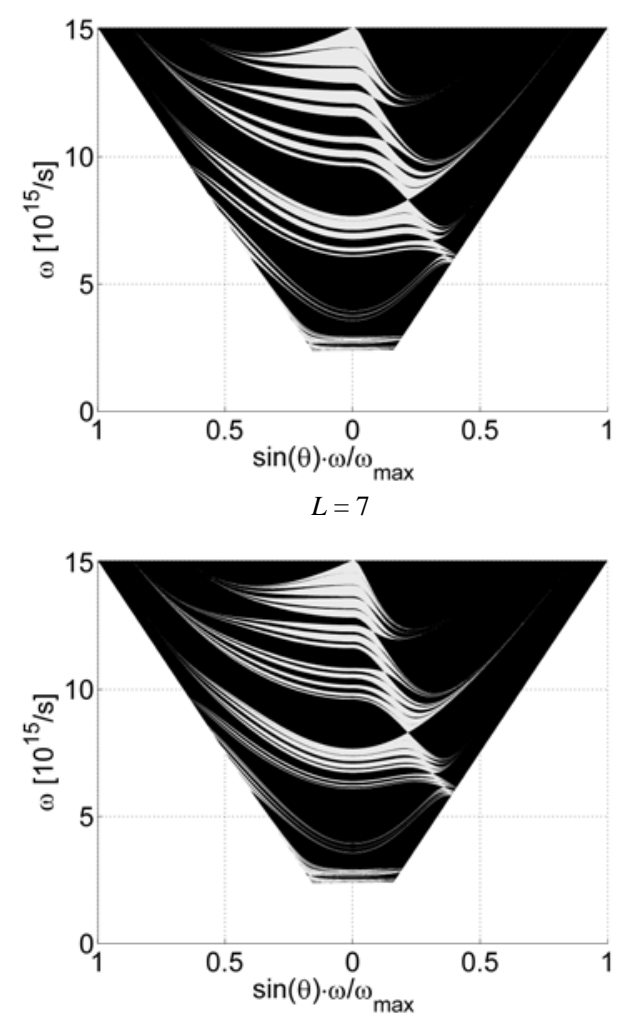

Fig. 3. Photonic band structure $\omega\left(k_{\perp}\right)$ for Fibonacci superlattices, $L$ is the generation number. reduces the solution to calculating $\tau$ and solving equation (3) by numerical algebraic methods.

The novelty of our research consists in applying this approach to superlattices made of strongly dispersive lossless metamaterial slabs.

The main results of this brief note are:

1. We observed a fractalization phenomenon of the Fibonacci superlattice photonic pass bands. We treat fractalization as the process of increasing the number of pass bands with the growing generation number $L$. Counting pass bands in the range of $5 \cdot 10^{15} \mathrm{~Hz}$ $15 \cdot 10^{15} \mathrm{~Hz}$ in Fig.4 one gets, for $L=4$, the number of pass bands $(K)$ equal to the Fibonacci number $\mathrm{F}_{4}=5$, and similarly for $L=5, K=\mathrm{F}_{5}=8, L=6, K=\mathrm{F}_{6}=13$, $L=7, K=\mathrm{F}_{7}=21$. The described phenomenon can be seen in both polarizations, although it is easier for polarization $\mathrm{p}$.

2. Our calculations confirm the existence of zero- $\bar{n}$ gaps in PBS of FS, which can be seen in Fig. 3 as the lowest (in frequency) band gaps.

3. Increasing the incidence angle (cf. Fig. 2.) decreases the number of photonic dispersion branches $\omega(q)$ in the investigated range $\left(\omega_{\mathrm{pm}}, \omega_{\max }\right)$. The highest dispersion branches are moved beyond $\omega_{\max }$.

\section{References}

[1] E. Macia, Rep. Prog. Phys. 69, 397 (2006)

[2] W. Steurer, D. Suter-Widmer, J. Phys. D: Appl. Phys. 40, R229 (2007).

[3] S. A. Ramakrishna, T. M. Grzegorczyk, Physics and Applications of Negative Refractive Index Materials, SPIE Press and CRC Press 2009

[4] J.E. Lugo et al, Optics Express, 17, 3042 (2009)

[5] H. Zhang, X. Chen, Y. Li, Y. Fu, N. Yuan, Optics Express, 17, 7800 (2009)

[6] M. de Dios-Leyva, J.C. Drake-Perez, Phys. Rev. E, 79, 036608 (2009)

[7] M. de Dios-Leyva, O.E. Gonzales-Vasquez, Phys. Rev. B, 77,125102 (2008)

[8] R. Srivastava, K.B. Thapa, S. Pati, S.P. Ojha, Sol. State Comm., 147, 157 (2008)

[9] Y. Fang, S. He, Phys. Rev. A, 78, 023813 (2008)

[10] A. Bruno-Alfonso, E. Reyes-Gomez, S.B. Cavalcanti, L.E. Oliveira, Phys. Rev. A, 78, 035801 (2008)

[11] Eds. C. M. Krowne, Y. Zhang, Physics of Negative Refraction and Negative Index Materials, Springer 2007

[12] Y.-T Fang, J. Zhou, E.Y.B. Pun, Appl. Phys. B, 86, 587 (2007)

[13] X. Hu, Z. Liu, Q. Gong, J. Opt. A: Pure Appl. Opt., 9, 877 (2007)

[14] F. F. de Medeiros, E. L. Albuquerque, M. S. Vasconcelos, Surface Science, 601, 4492 (2007)

[15] K. Tarnowski, W. Salejda, M. H. Tyc, Optica Applicata, 37, 387, 2007

[16] M.H. Tyc, W. Salejda, A. Klauzer-Kruszyna, K. Tarnowski, SPIE Proceedings Series, 2007, 6581, 658112; ibidem, 2007, 6581, 658113

[17] P. Yeh, Optical Waves in Layered Media, Wiley-Interscience 2005

[18] H.X. Da, C. Xu, Z.Y. Li, Phys. Lett. A, 345, 459 (2005)

[19] W. Salejda, A. Klauzer-Kruszyna, M. H. Tyc, K. Tarnowski, SPIE Proceedings Series, 2005, 5955, 595514; ibidem 2005, 5950, 59501Q

[20] J. Li, D. Zhao, Z. Liu, Phys. Lett. A, 332, 461 (2004)

[21] A. Klauzer- Kruszyna, W. Salejda, M.H. Tyc, Optik, 115, 257--266 (2004); ibidem 115, 267--276 (2004)

[22] M. Kohomoto, B. Sutherland, K. Iguchi, Phys. Rev. Lett., 58, 2436 (1987)

[23] J. Li, L. Zhou, C.T. Chan, P. Shang, Phys. Rev. Lett., 90, 083901 (2003) 